\title{
LEARNING EMBEDDINGS FOR SPEAKER CLUSTERING BASED ON VOICE EQUALITY
}

\author{
Yanick X. Lukic, Carlo Vogt, Oliver Dürr, and Thilo Stadelmann \\ Zurich University of Applied Sciences, Winterthur, Switzerland
}

\begin{abstract}
Recent work has shown that convolutional neural networks (CNNs) trained in a supervised fashion for speaker identification are able to extract features from spectrograms which can be used for speaker clustering. These features are represented by the activations of a certain hidden layer and are called embeddings. However, previous approaches require plenty of additional speaker data to learn the embedding, and although the clustering results are then on par with more traditional approaches using MFCC features etc., room for improvements stems from the fact that these embeddings are trained with a surrogate task that is rather far away from segregating unknown voices - namely, identifying few specific speakers.

We address both problems by training a CNN to extract embeddings that are similar for equal speakers (regardless of their specific identity) using weakly labeled data. We demonstrate our approach on the well-known TIMIT dataset that has often been used for speaker clustering experiments in the past. We exceed the clustering performance of all previous approaches, but require just 100 instead of 590 unrelated speakers to learn an embedding suited for clustering.
\end{abstract}

Index Terms - Speaker Clustering, Speaker Recognition, Convolutional Neural Network, Speaker Embedding

\section{INTRODUCTION}

Speaker clustering handles the "who spoke when" challenge in a given audio recording without knowing how many and which speakers are present in the audio signal. It is called speaker diarization when the task of segmenting the audio stream into speaker-specific segments is handled simultaneously [1]. The problem of speaker clustering is eminent in digitizing audio archives like e.g. recordings of lectures, conferences or debates [2]. For their quantitative indexing, automatic extraction of key figures like number of speakers or talk time per person is important. This further facilitates automatic transcripts using existing speech recognition procedures, based on the accurate automatic assignment of speech utterances to groups that each represent a (previously unknown) speaker.

The lack of knowledge of the number and identity of speakers leads to a much more complex problem compared to the related tasks of speaker verification and speaker identification, and in turn to less accurate results. One reason is that well-known speech features and models, originally fitted to the latter tasks, might not be adequate for the more complex clustering task [3]. The use of deep learning methods offers a solution [4]: in contrast to classical approaches (e.g., based on MFCC features and GMM models [5]), where general features and models are designed manually and independently for a wide variety of tasks, deep models learn hierarchies of suitable representations for the specific task at hand [6]. Especially convolutional neural networks (CNNs) have proven to be very useful for pattern recognition tasks mainly on images [7], but also on sounds [8]. Previous work [9] has shown that CNNs are able to learn a voice-specific vector representation (embedding) suitable for clustering when trained for the surrogate task of speaker identification. The authors report state of the art results for speaker clustering using an embedding learned from 590 different speakers.

In this paper, we investigate a novel training approach for CNNs for speaker clustering that learns embeddings more directly based on pairwise voice equality information of speech snippets (i.e., the binary information if the two snippets come from the same speaker or not). This weak labeling is neither a fitting to particular individuals, nor depending on hard to obtain voice similarity measures (i.e., real-valued distances amongst snippets). For evaluation, we focus on the pure speaker clustering performance, given its role as a performance bottleneck in the complete diarization process [3]. Section 2 reviews related work and introduces our approach. Section 3 reports on our results that not only reach state of the art consistently with one approach and improve the clustering quality in certain scenarios, but also reduce the necessary amount of pre-training data to $17 \%$. We also report on a number of experiments in order to give insight on which part of our system is responsible for the improved results. We conclude the paper with an outlook in section 4 .

\section{LEARNING SPEAKER DISSIMILARITY}

\subsection{Related work}

The design of CNNs makes it possible to recognize patterns in minimally preprocessed digital images or other data with 


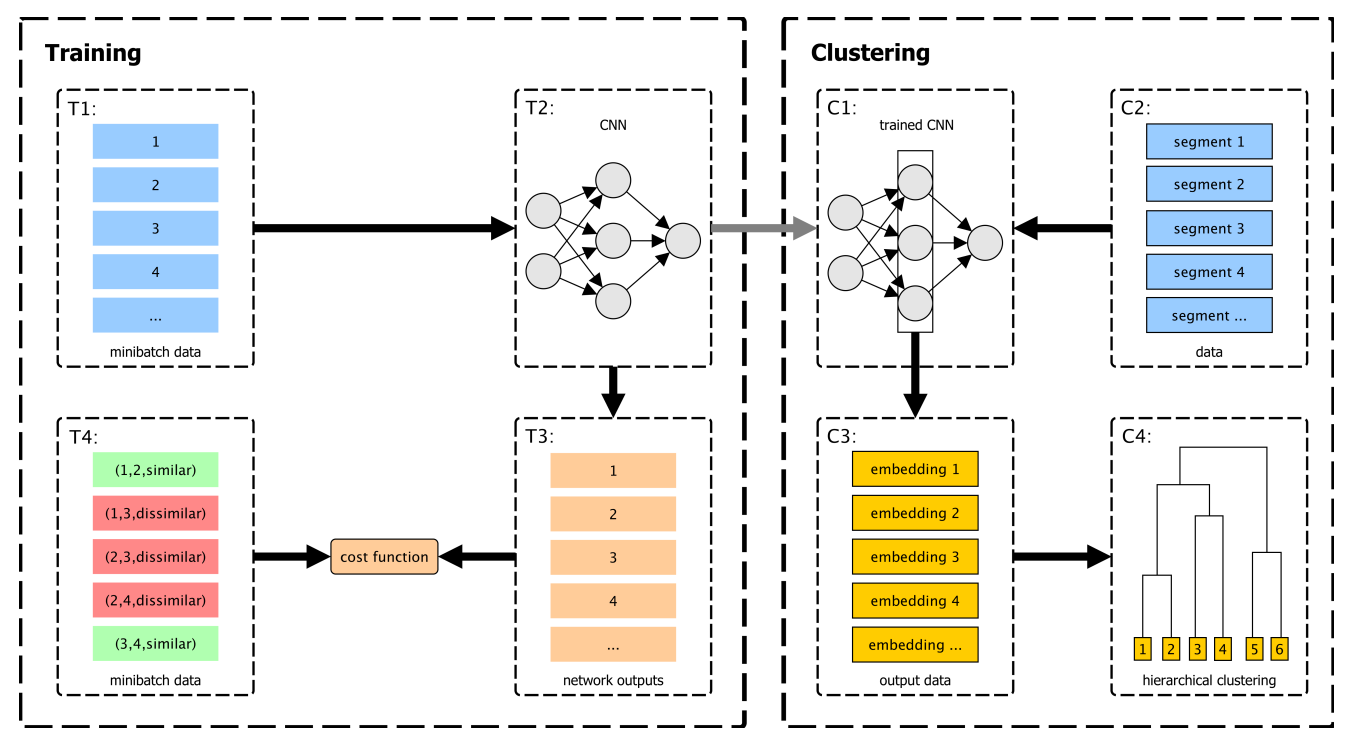

Fig. 1. Our overall speaker clustering process. Shown are the training steps (T1-T4) and the steps for the generation of speaker representations which are then hierarchically clustered $(\mathrm{C} 1-\mathrm{C} 4)$.

two dimensional structure by exploiting local connectivity. CNNs and other deep neural network (DNN) architectures thus have become the standard technique in many perceptual tasks [10][11][12][13]. Notable examples on audio data include Lee et al., who demonstrate how spectrograms can serve as inputs for a deep architecture in various audio recognition tasks [4]; and van den Oord and colleagues who build a generative model for sound (speech and otherwise) using a CNN variant [14]. Salamon et al. show how data augmentation serves to use CNNs for environmental sound classification, having only scarce data [15]. Milner and Hain attempt speaker diarization by iteratively re-labeling an initial set of utterances using DNNs per utterance that have been adapted from a single speaker recognition DNN, and then re-training the DNNs per resulting segment [16]. McLaren et al. address speaker recognition by using senone probabilities computed by a CNN, reaching results on par with an i-vector approach [17], and Sell et al. use a similar approach for speaker diarization [18]. However, previous work in the field of learning speaker characteristics using neural networks mostly relies on preprocessed features like MFCCs and doesn't use the feature learning capabilities of CNNs [19][20].

Recent advances have shown that it is possible to learn speaker-specific features from hidden layers of neural networks. Yella, Stolcke and Slaney used speaker-specific features derived from a 3 layer neural network together with MFCCs to subsequently feed them into a GMM/HMM system for speaker clustering [20]. This concept of speaker embeddings was further developed by Rouvier et al. through speaker representations derived from a non-convolutional neural network, using a super-vector obtained from a GMM-UBM as input vector [21].
It has been shown in [9] that CNNs are able to learn valuable features from spectrograms for speaker clustering. The authors use a single fully connected layer of a trained classification network to extract speaker embeddings (feature vectors) and subsequently cluster unknown speakers, achieving state of the art results. In contrast to this approach, we change the learning process fundamentally in this paper by using pairwise constraints instead of relying on a rather unrelated surrogate task for the creation of the speaker embeddings. This approach has been introduced in [22] using the MNIST dataset and was further developed in [23] to automatically discover image categories in unlabeled data. The process is similar in spirit to a siamese architecture [24], but building all possible pairs of snippets per mini-batch here allows for much more efficient training.

\subsection{Our approach}

Our approach consists of two major stages (see Fig. 1): training (T1-T4) and clustering $(\mathrm{C} 1-\mathrm{C} 4)$. The training stage is based on the work of Hsu and Kira [22]. Training is performed using mini-batch gradient descent, whereas the spectrograms are built exactly as in [9]. To attain a high diversification of the mini-batch composition (T1), for each member a random sentence from the training set is selected, and from this a random snippet of one second is used. A snippet has dimensionality $128 \times 100($ frequency $\times$ time $)$. We combine 100 such snippets in a mini-batch and let the network (T2) compute an output (T3) for each snippet in the minibatch. An output consists of the activations of a dense layer. Then, all possible pairs of these outputs are built, accompanied by weak labels stating whether the two original snippets 


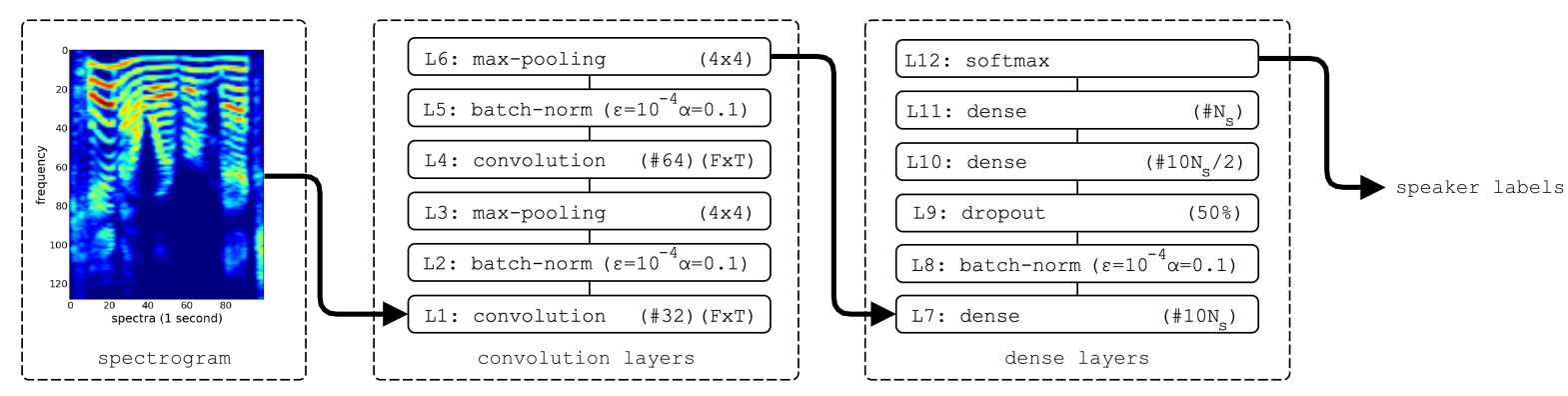

Fig. 2. The shown architecture is used for all experiments. In the convolutional layers, $F \times T$ corresponds to the dimension of the applied frequency $\times$ time filters.

are equal (same speaker) or not (different speakers) (T4). The error per pair is calculated by a cost function associated with the Kullback-Leibler divergence (KL divergence) to minimize the distance between equal pairs while maximizing the distance for unequal pairs [22]. This trains embeddings focused on voice equality and, by implication, similarity.

For a given pair of snippets $x_{p}$ and $x_{q}$, imagine that the network $f$ produces the embeddings $P$ and $Q$ of dimensionality $k$, respectively. These can be regarded as two discrete probability distributions $\mathbf{P}=f\left(x_{p}\right)$ and $\mathbf{Q}=f\left(x_{q}\right)$ over high-level voice-related features, for which the KL divergence is defined as follows:

$$
\mathrm{KL}(\mathbf{P} \| \mathbf{Q})=\sum_{i=0}^{k} P_{i} \log \left(\frac{P_{i}}{Q_{i}}\right)
$$

To use the KL divergence in the cost function, two indicator functions $I_{s}$ and $I_{d s}$ are introduced, where $I_{s}=1$ if $\left(x_{p}, x_{q}\right)$ is an equal pair w.r.t. voice, and $I_{d s}=1$ if it is a dissimilar pair, otherwise 0 . The hyperparameter margin in the hinge-loss part of the cost function sets the maximum margin between dissimilar pairs; we set it to 2 as proposed in [22].

$$
\begin{aligned}
& \operatorname{cost}(\mathbf{P} \| \mathbf{Q})=I_{s}\left(x_{p}, x_{q}\right) \cdot \mathrm{KL}(\mathbf{P} \| \mathbf{Q}) \\
& +I_{d s}\left(x_{p}, x_{q}\right) \cdot \max (0, \operatorname{margin}-\mathrm{KL}(\mathbf{P} \| \mathbf{Q}))
\end{aligned}
$$

The total cost $\mathrm{L}$ for the pair $\left(x_{p}, x_{q}\right)$ is calculated symmetrically via:

$$
\mathrm{L}(\mathbf{P}, \mathbf{Q})=\operatorname{cost}(\mathbf{P} \| \mathbf{Q})+\operatorname{cost}(\mathbf{Q} \| \mathbf{P})
$$

In the clustering stage, the trained network $(\mathrm{C} 1)$ is then used to generate embeddings from given speech utterances of unknown speakers (segments in C2). Utterances are split into non-overlapping 1s long snippets, then an embedding (C3) is extracted per snippet from one of the hidden layers (specified below) by using the activations of this layer. An embedding per utterance is formed by averaging over all embeddings of its snippets. Finally, as in [9], agglomerative hierarchical clustering (C4) with complete linkage is performed using the cosine distances between these mean embeddings of each utterance. We use the misclassification rate $(M R)$ as defined by
Kotti et al. [25] to evaluate the clustering performance in a way comparable to related work: A $M R$ of 0 confirms perfect assignment (all utterances of the same speaker in one cluster) and a $M R$ of 1 means that all utterances are assigned wrongly (falsely separated or mixed up in non-pure clusters). The $M R$ is defined as follows:

$$
M R=\frac{1}{N} \sum_{j=1}^{N_{c}} e_{j} .
$$

$N$ corresponds to the total number of utterances to be clustered, $N_{c}$ to the number of speakers (=clusters) and $e_{j}$ to the number of incorrectly assigned utterances of speaker $j$. The reported $M R$ for an experiment corresponds to the lowest $M R$ reached by varying the cutting point of the agglomerative hierarchical clustering manually (we thus ignore the problem of optimal cut point detection to focus on the task of clustering in an optimal order in accordance with [3]). The reported confidence intervals are calculated using Wilson method for a binomial probabilities with $2 \cdot N$ observations and $M R \cdot N$ successes.

Note that the proposed clustering method is not learned completely end-to-end. However, the surrogate task (learning to produce similar distributions for speech snippet pairs from the same speaker, based on short utterances) of the CNN is much closer to the final clustering task as compared to when training a standard speaker identification model to extract speaker embeddings (where the surrogate task is to classify specific voices, thus focusing on the characteristics of the concrete speakers $x_{1} . . x_{N_{c}}$ ). The learned embeddings therefore should be more suitable for clustering.

The architecture of our CNN is derived from [9] (compare Fig. 1, T2 and C1). We enhance it by using batch norm layers [26] after each convolutional layer and the first dense layer, and by changing the cost function as described above. Apart from that, the architecture stays the same. The detailed modified architecture is shown in Fig. 2. 


\section{EXPERIMENTAL SETUP AND RESULTS}

All experiments are carried out on the TIMIT dataset using the exact experimental setup as in previous work to be comparable [9][3]. Especially data splits and the used subsets of speakers for training and clustering follow these provisions closely. This means that when we perform clustering of $N_{c}$ speakers, we have overall $2 \cdot N_{c}$ utterances in our data set: two per speaker, where one is composed of the first 8 sentences of the speaker (lexicographically ordered by filename), and the other from the remaining two sentences, yielding an average utterance lengths of 20 and 5 seconds, respectively.

We carry out two major experiments: First, we test in a minimal setup whether it is possible to learn the distances (w.r.t. voice equality) between spectrograms. Second, we evaluate clustering performance on up to $N_{c}=80$ speakers, using our network pretrained on $N_{t}=100$ unrelated training speakers.

\subsection{Feasibility of KL divergence-related cost}

To assess whether our approach is able to learn the KL divergence-related cost function for two snippets, we train a network with $N_{t}=10$ speakers and then cluster $N_{c}=5$ and $N_{c}=10$ unrelated speakers on the activations of all layers separately. Thereby we can ascertain that the network is able to learn from the given constraints. Additionally, we can observe whether this cost formulation provides an advantage for speaker embedding extraction for clustering over the approach in [9]. We thus replicate as much of the training procedure from there (except the cost function) as possible, including training by stochastic gradient descent (SGD) with Nesterov momentum.

Performing the clustering experiment using $N_{c}=5$ unknown speakers we achieve a $M R$ of 0.0 on all layers. However, when looking at the t-SNE [27] (with cosine metric) plot per snippet in Fig. 3, we observe that the first dense layer L7 provides a much better visual clustering than the third dense layer L11. This is substantiated when performing the clustering experiment with $N_{c}=10$ unknown speakers: clustering on the third dense layer performs worse ( $M R$ of 0.3 ) than on the activations of both other dense layers, where a $M R$ of 0.1 is achieved. Thus, our speaker embeddings using the new similarity-based training regime clearly work, and seem to do so better if we extract them from lower dense layers.

\subsection{Improved optimization to cluster more speakers}

When increasing the complexity of the experiment by naively training a bigger network ( $N_{t}=100$ speakers $)$ and clustering more speakers $\left(N_{c}=40\right.$ speakers), the clustering performance decreases significantly $(M R=0.375)$. Hence, we try different additional optimization approaches using Adam [28], Adadelta [29] and, for reasons of comparability, also SGD without any momentum. We choose the algorithms'
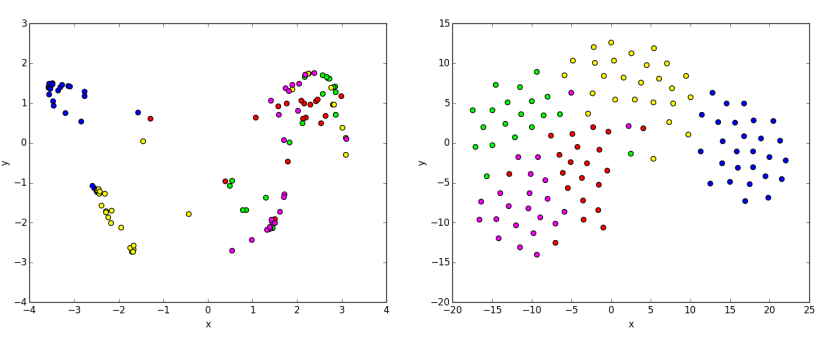

Fig. 3. Clustering quality visualization using t-SNE plots for embeddings extracted from the third dense layer L11 (left) and the first dense layer L7 (right). Different colors correspond to different speakers.

hyperparameters based on preceding experiments as follows: SGD with learning rate 0.001 ; Nesterov with learning rate 0.001 and momentum 0.9; Adam with learning rate 0.001, $\beta_{1}=0.9, \beta_{2}=0.999$ and $\epsilon=10^{-8}$; Adadelta with learning rate $1.0, \rho=0.95$ and $\epsilon=10^{-6}$. All resulting networks are trained for $10^{\prime} 000-30^{\prime} 000$ mini-batches (with mini-batch size $B=100$ ). The results for the clustering set of $N_{c}=40$ speakers are shown in Tab. 1.

SGD results in high $M R$ values, while Nesterov momentum performs better. More complex optimizers like Adam and Adadelta reach even better results. Additionally, they still show an upward trend after $10 \mathrm{~K}$ mini-batches. Therefore, we train these for additional mini-batches including all validation data. While Adam starts to show a performance decrease, Adadelta still improves for additional $10 \mathrm{~K}$ minibatches before leveling off on the best performing dense layer (L7).

\subsection{Embeddings of lower layers for better generalization}

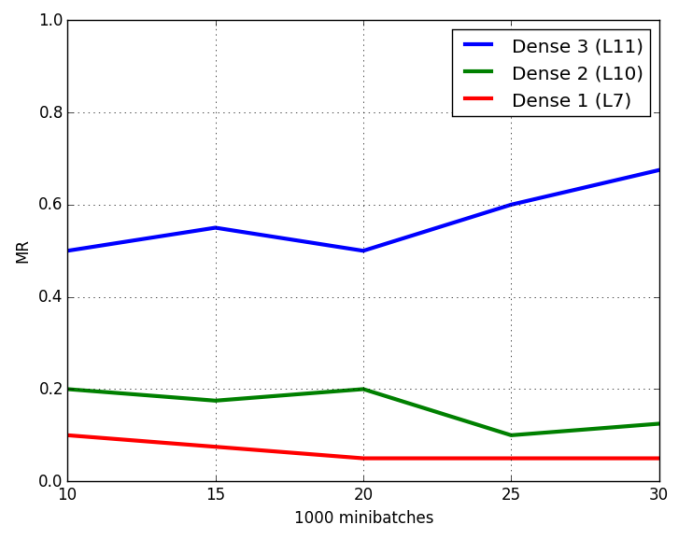

Fig. 4. Achieved $M R s$ for the different training stages (numbers of mini batches) of the Adadelta-trained network.

The MRs during training using Adadelta are shown in 
Table 1. Estimates and 0.95 Wilson-Confidence intervalls of $M R s$ for all training methods for $N_{c}=40$ unknown speakers

\begin{tabular}{l|llll|ll|l|l}
\hline Mini-batches $\rightarrow$ & $\mathbf{1 0 K}$ & & & & $\mathbf{1 5 K}$ & & $\mathbf{2 0 K}$ & $\mathbf{3 0 K}$ \\
\hline Layer $\downarrow$ & SGD & Nesterov & Adam & Adadelta & Adam & Adadelta & Adadelta & Adadelta \\
\hline L7 & $0.8_{-0.1}^{+0.17}$ & $0.6_{-0.11}^{+0.21}$ & $0.1_{-0.05}^{+0.13}$ & $0.08_{-0.04}^{+0.12}$ & $0.13_{-0.056}^{+0.15}$ & $0.08_{-0.04}^{+0.12}$ & $\mathbf{0 . 0 5}$ \\
L10 & $0.8_{-0.10}^{+0.18}$ & $0.38_{-0.01}^{+0.21}$ & $0.2_{-0.07}^{+0.17}$ & $0.18_{-0.07}^{+0.17}$ & $0.35_{-0.1}^{+0.2}$ & $0.08_{-0.04}^{+0.12}$ & $0.2_{-0.07}^{+0.17}$ & $0.13_{-0.06}^{+0.15}$ \\
L11 & $0.8_{-0.1}^{+0.17}$ & $0.6_{-0.11}^{+0.21}$ & $0.5_{-0.11}^{+0.21}$ & $0.55_{-0.11}^{+0.21}$ & $0.7_{-0.11}^{+0.2}$ & $0.38_{-0.1}^{+0.21}$ & $0.5_{-0.11}^{+0.21}$ & $0.68_{-0.11}^{+0.20}$ \\
\hline
\end{tabular}

Fig. 4. Interestingly, this network shows a performance decrease after $20 K$ mini-batches on the third dense layer L11: That is the same point where the performance on the first dense layer L7 becomes constant; and where the performance on the second dense layer L10 exhibits increased fluctuations without decreasing dramatically. We conjecture that this worsening performance of the higher layers is due to only the dense layers (as opposed to the convolutional layers) overadapting to the training data and the specific voices contained therein. A speaker representation directly built from the output of the last convolutional layer thus shouldn't be much affected by this, and lower dense layers only in weakened form. The same phenomenon is also observable when using Adam: while using higher layers for creating the embeddings significantly worsens the performance by $50 \%$ (L10) and $27 \%$ (L11) after additional $5 \mathrm{~K}$ mini-batches, performance on the first dense layer L7 only degrades by $17 \%$.

This assumed robustness of the convolutional layers to overadaptation is confirmed in an additional experiment: in this experiment, some of the speakers used to train the network (known speakers) are also used in the subsequent clustering alongside the usual unknown speakers that the network didn't encounter during training. The experiment is conducted using the Adadelta $20 K$ network that we deem overadapted to speaker identities encountered during training. While the results on the second (L10) and the third dense layer (L11) are better for the known speakers, the results on the first dense layer (L7) reach similar results for both known and unknown cases, indicating that convolutional layers are really less adapted to the particular identity of a speaker.

\subsection{Training on a more suitable task for more epochs to reach state of the art}

Overall, the best networks (Adadelta $20 K-30 K$ ) achieve a $M R$ of 0.00 and 0.05 for $N_{c}=20$ and $N_{c}=40$ speakers, respectively. This improves the results from [9] on the clustering set of 20 speakers by 10 percentage points and matches those of 40 speakers. We account this to the fact that training a network by learning distances between snippets corresponds closer to the actual clustering task than training on a surrogate classification task. Therefore better results are achieved with significantly less training data (100 vs. 590 speakers in the training set) than in the previous work of [9].

Table 2. Estimates and 0.95 Wilson-Confidence intervalls of $M R s$ for our best-performing models

\begin{tabular}{l|lll}
\hline Method & $N_{c}=20$ & 40 & 80 \\
\hline Adadelta $20 K$ & $0_{-0}^{+0.09}$ & $0.05_{-0.03}^{+0.10}$ & $0.18_{-0.05}^{+0.12}$ \\
Adadelta $30 K$ & $\mathbf{0 . 0}_{-0}^{+0.09}$ & $\mathbf{0 . 0 5}_{-0.03}^{+0.03}$ & $\mathbf{0 . 1 4}+0.05$ \\
CNN [9] & $0.1_{-0.06}^{+0.2}$ & $0.05_{-0.03}^{+0.10}$ & - \\
$\nu$-SVM [3] & - & 0.06 & - \\
GMM/MFCC [3] & 0.0 & $0.13_{-0.06}^{+0.15}$ & - \\
\hline
\end{tabular}

Tab. 2 gives a comparison of our best results (Adadelta $20 K-30 K$ ) with other methods from the literature that use the same experimental setup. Going beyond the state of the art in clustering more than 40 speakers indicates that effective training happens even beyond the point of the leveling off of the objective function: the Adadelta $30 \mathrm{~K}$ network clearly outperforms the one trained for only $20 \mathrm{~K}$ mini-batches when clustering 60 (not shown) or even 80 speakers. Regarding computational performance, all methods except the $\nu$-SVM approach of [3] perform clustering ca. in real-time (after offline training).

\section{CONCLUSIONS AND FUTURE WORK}

This paper proposes to perform speaker clustering based on embeddings extracted from the lowest dense layer of a CNN, trained to learn a distance between speech snippets with respect to voice equality using only binary pairwise constraints (same/different speaker) on pre-training snippets. In terms of misclassification rate, our approach is on par with the state of the art in clustering performance for 40 speakers [9] and 20 speakers [3]. However, our model is more robust in the sense that it (a) achieves this result with $83 \%$ less pre-training data as compared to the other deep learning approach [9], and (b) reaches state of the art consistently for both speaker set sizes, with a single approach. It goes beyond the state of the art in also clustering 60 and 80 speakers reasonably well ( $M R=0.13)$ on the TIMIT task, which to our best knowledge has never been reported before.

Future work will investigate how to explicitly learn to cluster speakers given only utterances and therefore develop 
our approach to become an entirely end-to-end learning task without a downstream agglomerative hierarchical clustering process. On the short run, using an explicit embedding layer as in [21] could grant better results. Also, the convolutional filters of the network show many duplicates; therefore the model could likely be improved by reducing the network size or by reinitializing the duplicate filters throughout the training with noise. Finally, the effect of more diverse background speaker data on the number of clusterable speakers seems worth exploring.

\section{REFERENCES}

[1] Beigi, Fundamentals of speaker recognition, Springer Science \& Business Media, 2011.

[2] Anguera, Bozonnet, Evans, Fredouille, Friedland, and Vinyals, "Speaker diarization: A review of recent research," IEEE Trans. ASLP, vol. 20, no. 2, pp. 356-370, 2012.

[3] Stadelmann and Freisleben, "Unfolding speaker clustering potential: a biomimetic approach," in Proc. ACM Multimedia, 2009, pp. 185-194.

[4] Lee, Pham, Largman, and Ng, "Unsupervised feature learning for audio classification using convolutional deep belief networks," in Adv. NIPS, 2009, pp. 10961104.

[5] Reynolds, "Speaker identification and verification using gaussian mixture speaker models," Speech Communication, vol. 17, no. 1, pp. 91-108, 1995.

[6] Bengio, Courville, and Vincent, "Representation learning: A review and new perspectives," IEEE Trans. PAMI, vol. 35, no. 8, pp. 1798-1828, 2013.

[7] LeCun, Bengio, and Hinton, "Deep learning," Nature, vol. 521, no. 7553, pp. 436-444, 2015.

[8] Yu and Deng, Automatic Speech Recognition, Springer, 2012.

[9] Lukic, Vogt, Dürr, and Stadelmann, "Speaker identification and clustering using convolutional neural networks," in Proc. IEEE MLSP, 2016, pp. 1-6.

[10] Schmidhuber, "Deep learning in neural networks: An overview," Neural Networks, vol. 61, pp. 85-117, 2015.

[11] Szegedy, Liu, Jia, Sermanet, Reed, Anguelov, Erhan, Vanhoucke, and Rabinovich, "Going deeper with convolutions," in Proc. IEEE CVPR, 2015, pp. 1-9.

[12] Krizhevsky, Sutskever, and Hinton, "Imagenet classification with deep convolutional neural networks," in $A d v$. NIPS, 2012, pp. 1097-1105.

[13] LeCun and Bengio, "Convolutional networks for images, speech, and time series," The handbook of brain theory and neural networks, vol. 3361, no. 10, pp. 1995, 1995.
[14] van den Oord, Dieleman, Zen, Simonyan, Vinyals, Graves, Kalchbrenner, Senior, and Kavukcuoglu, "Wavenet: A generative model for raw audio," CoRR, vol. abs/1609.03499, 2016.

[15] Salamon and Bello, "Deep convolutional neural networks and data augmentation for environmental sound classification," IEEE Signal Processing Letters, vol. 24, no. 3, pp. 279-283, 2017.

[16] Milner and Hain, "Dnn-based speaker clustering for speaker diarisation," in Proc. Interspeech, 2016, pp. 2185-2189.

[17] McLaren, Lei, Scheffer, and Ferrer, "Application of convolutional neural networks to speaker recognition in noisy conditions.," in Proc. INTERSPEECH, 2014, pp. 686-690.

[18] Sell, Garcia-Romero, and McCree, "Speaker diarization with i-vectors from dnn senone posteriors," in Proc. Interspeech, 2015, pp. 3096-3099.

[19] Chen and Salman, "Learning speaker-specific characteristics with a deep neural architecture," IEEE Trans. Neural Networks, vol. 22, no. 11, pp. 1744-1756, 2011.

[20] Yella, Stolcke, and Slaney, "Artificial neural network features for speaker diarization," in IEEE SLT Workshop. IEEE, 2014, pp. 402-406.

[21] Rouvier, Bousquet, and Favre, "Speaker diarization through speaker embeddings," in Proc. EUSIPCO. IEEE, 2015, pp. 2082-2086.

[22] Hsu and Kira, "Neural network-based clustering using pairwise constraints," CoRR, vol. abs/1511.06321, 2015.

[23] Hsu, Lv, and Kira, "Deep image category discovery using a transferred similarity function," CoRR, vol. abs/1612.01253, 2016.

[24] Chen and Salman, "Extracting speaker-specific information with a regularized siamese deep network," in Adv. NIPS, 2011, pp. 298-306.

[25] Kotti, Moschou, and Kotropoulos, "Speaker segmentation and clustering," Signal Processing, vol. 88, no. 5, pp. 1091-1124, 2008.

[26] Ioffe and Szegedy, "Batch normalization: Accelerating deep network training by reducing internal covariate shift," CoRR, vol. abs/1502.03167, 2015.

[27] van der Maaten and Hinton, "Visualizing data using tsne,” JMLR, vol. 9, no. Nov, pp. 2579-2605, 2008.

[28] Kingma and Ba, "Adam: A method for stochastic optimization," CoRR, vol. abs/1412.6980, 2014.

[29] Zeiler, "Adadelta: An adaptive learning rate method," CoRR, vol. abs/1212.5701, 2012. 